\title{
perifèria
}

Número 6, julio 2007

www.periferia. name

\section{Un largo "igar"}

Mónica Martínez Mauri - Universidad Autónoma de Barcelona - École des Hautes Études en Sciences Sociales ${ }^{1}$

\section{Resumen}

En este texto describo el largo camino que he recorrido desde el primer día que pensé trabajar con los kunas hasta la realización del trabajo de campo. Presento los motivos que me llevaron a Kuna Yala (Panamá) para obtener los permisos de investigación de las autoridades tradicionales indígenas (el Congreso General de la Cultura Kuna) y reflexiono sobre cómo la interacción con los congresistas, caciques, intelectuales y comuneros recondujeron los objetivos iniciales de la investigación durante los trabajos de campo realizados del 2000 al 2004.

Palabras clave: trabajo de campo, Kuna Yala, Panamá.

\section{Abstract}

In this paper I describe the long way that I have undertake when I decided to work among the kuna people of Panama until the realization of fieldwork. I present the reasons that carry me to Kuna Yala to obtain the research permits from the traditional authorities (General Congress of Kuna Culture) and I explain how the interaction with representatives, chiefs, intellectuals and commoners has changed the goals of this research during the fieldwork I carry out during the period 20002004.

Keywords: fieldwork, Kuna Yala, Panama.

En lengua kuna la palabra igar $^{2}$ corresponde con la idea de camino: un espacio bidireccional por donde circulan personas, animales, pero por donde también transitan ideas e historias de generación en generación. En cierta manera el concepto igar puede asemejarse con el camino que recorre un estudiante de antropología social durante la elaboración de una tesis doctoral. Un camino que lo transporta física y mentalmente a otras realidades, pero en el que también modifica sus pensamientos y construye nuevos conocimientos.

\footnotetext{
${ }^{1}$ Enviar correspondencia a: Mònica Martínez Mauri mommauri@yahoo.com; monica.martinez@uab.cat

${ }^{2}$ La palabra igar es sinónima de camino, historia, relato, canto. Su uso es parecido a la noción inglesa "way".
} 


\title{
perifèria
}

\author{
Número 6, julio 2007
}

www. periferia. name

En mi caso, los estudios de doctorado me han llevado a trabajar con el pueblo kuna

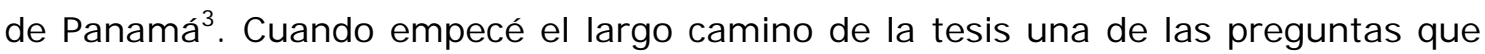
más me formularon mis amigos, colegas, familiares y "sujetos de estudio" fue: "¿por qué te interesaste por los kunas?" Afortunadamente, aunque los motivos y las ideas que me llevaron a Kuna Yala en 1999 se han ido modificando con el tiempo, nunca he dejado de encontrar nuevas respuestas a este interrogante.

Antes de describir estos motivos, debo reconocer que si no hubiera tenido que justificar repetidamente mi curiosidad por Kuna Yala, no recordaría como ha ido cambiando mi interés por esta sociedad -con la que todavía trabajo y convivo- ni hubiera elaborado un discurso coherente sobre las razones que me llevaron a esta particular región del planeta. La verdad es que la primera vez que oí a hablar de Kuna Yala, simplemente me sentí irracionalmente atraída por esta comarca autónoma en la que los indígenas controlaban el acceso a sus tierras, el turismo y la inversión extranjera. Fue un amor a primera vista. En ese momento todavía no buscaba un sitio de estudio con el que casarme, tan sólo me interesaba todo lo que era nuevo o desconocido. Así que en ese momento todo quedó en un flechazo. En ese entonces estaba a punto de terminar mis estudios de licenciatura en la UAB y no me planteaba seguir con el doctorado. Como no tenía recursos para aventurarme a empezar una tesis era inimaginable que pudiera viajar a Panamá para empezar una investigación etnográfica. Pero como bien canta el panameño Rubén Blades, a veces "la vida te da sorpresas": en 1999 "la Caixa" me concedió una beca para continuar mi formación en Francia. Viendo las nuevas posibilidades que me brindaba esta ayuda, no me lo pensé dos veces. Empecé a leer sobre Kuna Yala ${ }^{4}$, planteé una investigación de DEA en la Ecole des Hautes Etudes en Sciences

\footnotetext{
${ }^{3}$ Los kunas, junto a los ngobes, los wounaan, los emberas, los nasos, los buglés y los bri-bris, son uno de los siete pueblos indígenas que alberga el territorio panameño. Según las cifras del último censo (Contraloría General de la República de Panamá, 2000) son 61.707 y constituyen el segundo grupo indígena del país.

${ }^{4}$ Debo confesar que la etapa de lectura no fue nada fácil. Cuando en 1999 consulté por primera vez los Human Relation Area Files (HRAF) para localizar fuentes bibliográficas sobre la zona y sus gentes, me encontré con nada más ni nada menos que 844 referencias. Teniendo en cuenta que desde aquel
} 


\title{
perifèria
}

\author{
Número 6, julio 2007
}

www. periferia. name

Sociales (EHESS) y viajé a Panamá. Fue entonces cuando fui construyendo un discurso que justificase mi irracional interés por Kuna Yala.

La justificación racional de mi enamoramiento por Kuna Yala se fue centrando en confirmar que la experiencia kuna, gracias a sus particularidades históricas y su situación geopolítica, era excepcional para reflexionar sobre el alcance de las políticas de desarrollo sostenible y la participación indígena en el sistema de las Naciones Unidas. De hecho, de entre todas las razones que me llevaron a Kuna Yala, la autonomía política que habían logrado sus dirigentes durante la primera mitad del siglo XX me había cautivado por completo. Me sorprendía que un pueblo indígena hubiera podido negociar con el Estado panameño un tema tan espinoso como la autonomía política y reconozco que me dejaba atónita que todavía hoy se respetaran los acuerdos firmados 50 años atrás. Esta fascinación por su autonomía es lo que primero articuló mi proyecto de investigación centrado en los kunas. El hecho de que ellos mismos pudieran impedir que las multinacionales pusieran los pies en su territorio o que el Estado instalara una base naval dentro de su jurisdicción, me llevó a pensar que era el marco ideal para observar las dinámicas que acompañan la definición y ejecución de proyectos de desarrollo. Poco a poco me fui dando cuenta que era el único lugar en América Latina donde un pueblo indígena controlaba el acceso de ONG y proyectos de desarrollo a su región. Además, el Congreso General Kuna (CGK, el máximo órgano de decisión del pueblo kuna) había demostrado ser una instancia fuerte y consolidada tanto a escala nacional como internacional. No es de extrañar que, con el tiempo, la organización socio-política kuna se convirtiera en un modelo a seguir por otros pueblos indígenas, y que incluso recibiera reconocimientos internacionales, como el Premio Bartolomé de las Casas del Gobierno español en 1998.

entonces he recopilado fuentes que no estaban contempladas en los HRAF y se han publicado nuevos trabajos, se puede afirmar que, entre tesis, manuscritos no publicados, ponencias, artículos y libros, existen más de mil referencias sobre los kunas. Ante esta inquietante evidencia para una investigadora neonata como yo - pues es fácil pensar que ya todo se ha escrito sobre los kunas- me parecía imposible aportar mi granito de arena. 


\section{perifèria}

Número 6, julio 2007

www. periferia. name

A parte de la autonomía, mi interés por Kuna Yala también se justificaba con el éxito y reconocimiento internacional que obtuvo el Plan de Estudio y Manejo de las Áreas Silvestres de Kuna Yala, más conocido por sus siglas: PEMASKY, el primer proyecto de desarrollo sostenible y de conservación que formularon los kunas con la ayuda de organizaciones internacionales. Gracias a esta iniciativa, el pueblo kuna se convirtió en el primer grupo indígena que consiguió demarcar, proteger y gestionar un área forestal de 60.000 hectáreas. Con el PEMASKY, los kunas volvieron a ser un pueblo ejemplar. Durante la década de los ochenta, muchos cooperantes, científicos y estudiantes visitaron Nusagandi, la estación biológica que los kunas construyeron en medio de la selva. Querían ver con sus propios ojos cómo las concepciones tradicionales de utilización del bosque se combinaban con los avances científicos en materia de conservación. La resonancia que tuvo el proyecto llegó a ser impresionante. De hecho, la primera vez que oí hablar de los kunas fue a través del PEMASKY, cuando estudiaba antropología en la Universidad de Costa Rica y cayó en mis manos un artículo del director del proyecto que me fascinó 5 .

Después de constatar que los kunas también eran ejemplares en el campo de la conservación medioambiental, me di cuenta de que el pueblo kuna contaba con una buena plantilla de profesionales y técnicos indígenas que a través de sus organizaciones, normalmente ONG, participaban regularmente en las reuniones de Naciones Unidas dedicadas a los pueblos indígenas. Varios representantes kunas seguían los debates del Grupo de Trabajo sobre los Pueblos Indígenas en Ginebra y recorrían el mundo de conferencia en conferencia. Esto también alentó mi pasión por este pueblo. Mi curiosidad no paraba de crecer: ¿Cómo un pequeño pueblo indígena de Panamá podía conseguir una representación tan amplia?

Una vez demostré que tenía razones de peso para plantear una nueva tesis sobre Kuna Yala, accedí a los estudios de tercer ciclo en la École des Hautes Études en Sciences Sociales con el objetivo de estudiar el impacto de las políticas

\footnotetext{
${ }^{5}$ El artículo en cuestión se titulaba "PEMASKY en Kuna Yala: Protegiendo a la madre Tierra... y a sus hijos" escrito por Guillermo Archibold en 1993.
} 


\title{
perifèria
}

\author{
Número 6, julio 2007
}

www. periferia. name

internacionales en materia de desarrollo y derechos indígenas sobre la organización sociopolítica kuna. Con el tiempo, los objetivos e hipótesis que en un principio motivaron mi investigación doctoral se fueron transformando. Pronto observé que para llegar a entender los programas de desarrollo, el cambio social, la percepción del medio ambiente y el discurso político kuna, un largo trabajo de campo en Kuna Yala era necesario, pero no suficiente. También tenía que visitar las oficinas de las ONG kunas en la capital y establecer contactos con los directores de proyectos que pasaban más tiempo en la ciudad o en el extranjero que en sus comunidades de origen. Quería recoger el discurso político de los líderes kunas en los espacios de mediación, pero el Gobierno panameño y las agencias internacionales llegaban a las comunidades en contadas ocasiones. Si tenía la intención de investigar el papel y los efectos de las ONG y las políticas de desarrollo, era necesario viajar a Ginebra, ciudad de Panamá y Kuna Yala. En definitiva, después de seguir a los kunas durante dos años en Ginebra, en Kuna Yala y Panamá, los objetivos iniciales de mi investigación se fueron renovando y transformando consciente e inconscientemente.

\section{De cómo los kunas modelaron la investigación}

El año 2000 empecé una nueva etapa del largo camino de la tesis: el trabajo de campo. En Kuna Yala las investigaciones de campo siempre fueron concebidas desde una perspectiva clásica. Mi objetivo era emprender una etnografía a la vieja usanza, por ello conviví con una familia y una comunidad kuna, aprendiendo a hablar, dormir, comer, caminar... como ellos y ellas. El trabajo de campo en Kuna Yala y Panamá se realizó en cuatro momentos distintos: dos meses en 2000, tres en 2001, un año entre $2002-2003^{6}$ y tres meses en 2004. Para no dejar lugar a dudas, durante el período 2000-2004, pasé un total de 378 días en Kuna Yala (la mayor parte del tiempo en la comunidad de Gardi Sugdup) y 167 días en la ciudad de Panamá.

\footnotetext{
${ }^{6}$ Durante este año me acompañó mi pareja, Xavier. El resto de las estadías las realicé en solitario.
} 


\title{
perifèria
}

\author{
Número 6, julio 2007
}

www.periferia. name

Pero, a pesar de que todo fue enfocado desde una perspectiva clásica, a diferencia de las generaciones de antropólogos que me habían precedido en la región, antes de llegar a la comunidad y empezar con la clásica observación participante tuve que negociar la obtención del permiso de investigación con el Estado, el Congreso General de la Cultura Kuna y la comunidad. Aunque muchos pueden pensar que conseguir la autorización de las organizaciones indígenas no es necesario o que es cosa fácil, no hay que minimizar su importancia ni menospreciar sus mecanismos de decisión. La investigación de campo no puede empezar sin el permiso y el o la investigador/a debe adaptarse al tempo que marcan los órganos socio-políticos del pueblo con el que quiere trabajar. En mi caso tuve que entender como funcionaba el sistema de congresos.

Los kunas cuentan con dos congresos generales: el Congreso General Kuna (CGK), de carácter político-administrativo, y el Congreso General de la Cultura Kuna (CGCK), dedicado a los temas culturales y espirituales. Tanto el CGK como el CGCK están integrados por delegados y sailas (autoridades tradicionales) de las 48 comunidades que conforman la comarca de Kuna Yala y, normalmente, se reúnen dos veces al año. Después de interesarme por el funcionamiento del sistema, me confirmaron que para poder llevar a cabo mi proyecto de investigación tenía que contar con la autorización del CGCK. El secretario del Congreso me remitió a los artículos del Estatuto y la Ley Fundamental de la Comarca de Kuna Yala relacionados con la realización de estudios en las comunidades:

Estatuto de Kuna Yala, 1995: Art. 12: En caso de que el Congreso General de la Cultura Kuna concediere la autorización para cualquiera actividad investigativa a los no-kunas, sea ella, natural o jurídica, todo el proceso de estudio se llevará a cabo con la participación activa de uno o dos delegados nombrados por el mismo Congreso General de la Cultura, quienes deberán dar informes detallados de la misma a la Asamblea.

Las condiciones, los límites de investigación o de exploración los pondrá el Congreso General de la Cultura Kuna en coordinación con el Congreso General Kuna y el Congreso Local, sede del estudio. 


\section{perifèria}

Número 6, julio 2007

www. periferia. name

Ley Fundamental de la Comarca de Kuna Yala, 1995: Sobre investigaciones, Artículo 39: Para realizar investigaciones dentro de la Comarca, toda persona natural o jurídica interesada deberá contar con la autorización expresa del Congreso General de la Cultura Kuna, quedando obligada a entregar al mismo, un ejemplar del original de los resultados de la investigación en español.

El Congreso mencionado podrá exigir los beneficios de la misma, además de los derechos de propiedad intelectual y patentización, y reservarse el derecho de impedir y de suspender ciertas investigaciones, así como la divulgación de sus resultados que pueden ir contra la integridad del Pueblo Kuna.

Tantos los procedimientos como las condiciones para estas investigaciones serán fijadas en el Estatuto de la Comarca.

En 2001, conociendo la Ley y las reuniones que tenía programadas el CGCK, me introduje en la agenda de un congreso para presentar personalmente mi propuesta ante los, aproximadamente, 300 delegados, sailas y caciques. Antes de llegar a la asamblea busqué el asesoramiento de los intelectuales indígenas. Sus consejos me fueron muy útiles y sugerentes. Gracias a ellos pude adecuar la propuesta inicial a los intereses y preocupaciones de las autoridades de la comarca. De hecho, cuando conseguí presentar el proyecto ante el congreso me di cuenta que ya había realizado un mini-trabajo de campo para ajustar mis ideas surgidas entre Paris, Ginebra y Barcelona al contexto kuna y panameño. La verdad es que aprendí mucho durante las negociaciones.

Con todo, mi presentación ante el congreso fue un éxito. Después de responder algunas dudas, los congresistas me hicieron saber que la propuesta era del interés de las autoridades y que si me comprometía a cumplir con los artículos de la ley fundamental y del estatuto de Kuna Yala no tendría ningún problema en las comunidades. Para cerrar el acuerdo y reforzar mi compromiso me exigieron una carta de uno de mis directores de tesis conforme entendía las normas marcadas por el congreso. 


\title{
perifèria
}

\author{
Número 6, julio 2007
}

www. periferia. name

Como puede deducirse de esta explicación, aunque el proceso es sumamente instructivo, el principal problema de esta etapa exploratoria es el tiempo. Se necesita mucho tiempo y paciencia para conseguir el permiso de investigación, tener en cuenta las observaciones de los intelectuales locales y formular una propuesta adaptada a contextos completamente diferentes. Aunque debo reconocer que cuando me lo concedieron me sentí segura y en confianza, tardé más de un año para obtener el permiso del congreso.

Además, por si fuera poco, las negociaciones no terminaron con la concesión del permiso por el CGCK. Tras obtener la autorización de las autoridades kunas tuve que pedir el permiso de la Dirección General del Patrimonio Histórico de Panamá, pues para realizar investigaciones antropológicas en suelo panameño en última instancia se necesita el visto bueno del Estado. Este trámite es meramente formal, está completamente condicionado al consentimiento de las organizaciones indígenas. Este hecho reafirma la autonomía política del pueblo kuna, pues en la comarca, el papel que cuenta no es el del Estado, sino el de la organización indígena.

Finalmente, tuve que pedir permiso a la comunidad donde pensaba realizar mi trabajo de campo de larga duración: Gardi Sugdup, una pequeña isla del sector occidental de la comarca. En la comunidad también fue fundamental estar en posesión del permiso del congreso general. Cuando los sailas y los comuneros vieron que contaba con la aprobación de las autoridades de la comarca no tuve problemas para tener su visto bueno. Aceptaron que me quedara a vivir en el pueblo. Esta fue la primera vez que pude comprobar que Gardi, a diferencia de algunas comunidades de la comarca, respetaba y acataba las directrices de los congresos. Pero, aún y la confianza que habían depositado en mi, preferí exponer mi proyecto de investigación ante el congreso local.

El congreso local se reúne cada noche en la onmaket nega (casa del congreso). Está integrado mayoritariamente por hombres que discuten todos los problemas y proyectos que atañen a la colectividad. La casa del congreso es como el corazón de la comunidad, pues es donde late la vida social. Aprovechando una de estas reuniones, me presenté y expliqué que estaba ahí para estudiar el impacto de las 


\title{
perifèria
}

\author{
Número 6, julio 2007
}

www.periferia. name

políticas de desarrollo ideadas por las agencias internacionales en la comarca, que quería entender qué tipo de relación material y simbólica mantenían con los recursos (tanto acuáticos como terrestres), etc. Aunque al principio fui un poco escéptica, pronto me di cuenta de que este ejercicio me aportó mucho. Por un lado me ahorró malentendidos con los comuneros. Por el otro, me proporcionó informaciones muy relevantes -consensuadas por casi todos los hombres del pueblo- sobre los temas que había seleccionado. Vista esta primera experiencia, repetí la práctica para que la gente entendiera qué estaba buscando, me hiciera comentarios y viera como evolucionaba mi pensamiento. $\mathrm{Y}$ al final, siguiendo el modelo de los informes que las instituciones locales (cafetería, motonave, teléfonos públicos, dormitorio, planta eléctrica) entregaban al pleno, decidí yo también presentar un informe mensual para comentar mis avances a la comunidad. Aunque a veces parecía que rindiera cuentas al pueblo sobre mi trabajo, de esta manera la gente logró entender mi presencia en la comunidad y me ahorré posibles malentendidos.

\section{La investigación de campo día a día}

Como en la mayoría de trabajos de campo etnográficos, al principio no fue nada fácil adaptarse a las condiciones sociales y materiales de vida del pueblo. Las reducidas dimensiones de la isla (2,5 hectáreas) y la falta de un espacio propio, me provocaban una terrible sensación de claustrofobia y angustia. Poco a poco, el tiempo puso remedio a los primeros problemas de adaptación. Pronto empecé a entender la lengua y a comunicarme con las mujeres de la familia, me acostumbré a la comida, la hamaca y a mi nueva vida pública. De hecho en Gardi no había ni espacio ni tiempo para la intimidad, o sea que no tenía vida privada, mi vida era pública 24 horas al día.

Pero a pesar de la adaptación, los permisos, el respeto de las normas de la comunidad y la presentación de resultados ante el pleno del congreso una vez al mes, algunos ancianos de la isla, con los que supuestamente tenía que trabajar, no me veían con buenos ojos. Durante mis primeros meses en Gardi se mostraron reticentes a colaborar con la investigación. Según ellos, los antropólogos robaban la 


\title{
perifèria
}

\author{
Número 6, julio 2007
}

www.periferia. name

cultura, utilizaban al pueblo para hacerse ricos y no compartían los resultados de sus investigaciones con ellos.

No era la primera vez que la gente de Gardi Sugdup acogía a una antropóloga, pero como ya había podido percibir en la ciudad a través de mis contactos con los intelectuales kunas, este hecho me trajo más problemas que ventajas. Antes de ir a Kuna Yala ya sabía que los antropólogos no despertaban la simpatía de los locales. Había leído en la monografía de Karen Tice (1995) que durante los años ochenta y noventa los kunas habían rechazado, e incluso, expulsado a antropólogos de sus comunidades. También hacía poco que había descubierto que algunos de mis colegas ocultaban su oficio y se presentaban como cooperantes o sociólogos ante las personas con las que trabajaban por miedo a la mala reputación de nuestro gremio. Pero todas estas evidencias no me condujeron a ocultar mi profesión. En todos los contextos en los que trabajé siempre me presenté como antropóloga social. Como mi vida era totalmente pública y tenía que ser transparente para demostrar que era alguien de confianza, no me sentía cómoda ocultando algo tan importante como mi identidad profesional. Así que hice todo lo contrario, preferí avanzar de antemano mi condición de antropóloga con la ilusión de cambiar algunos de los estereotipos, a veces merecidos, de nuestra disciplina. A mi entender, la gente de Gardi Sugdup tenía que darse cuenta de que un estudio sobre su cultura también podía ser relevante para su futuro. Por eso les insistía en que, en lugar de cerrarse en balde, debían intervenir en la investigación, sugiriendo temas prioritarios a los interesados en estudiar algún aspecto de su sociedad o cultura, y exigiendo tanto permisos como resultados a los antropólogos que realizaban trabajos de campo en sus comunidades.

\section{La investigación etnográfica hoy: entre la academia y el campo}

Practicar la etnografía en la América indígena de hoy implica tener en cuenta los prejuicios hacia los antropólogos y hacer todo lo posible por cambiarlos. Creo que sólo de esta manera la antropología podrá servir a los intereses tanto de las poblaciones locales de la que se nutre, como de la academia. Lamentablemente lo expuesto hasta aquí denota que no es difícil adecuar los objetivos de la 


\title{
perifèria
}

\author{
Número 6, julio 2007
}

www. periferia. name

investigación a las necesidades de la gente con la que trabajamos.

Aunque, como recuerda Eric Fassin (2007), al igual que las otras ciencias sociales, la antropología siempre está implicada - pues nunca está al margen de la sociedada veces no sabemos muy bien de qué lado estamos. Olvidamos que nos servimos de una ciencia social que toma la sociedad como objeto y que, al mismo tiempo, está enraizada en la sociedad. Por ello, a mi entender, nuestra disciplina se encuentra con el problema de producir conocimientos en el lenguaje de la sociedad de acogida. Es como si hubiéramos construido una discontinuidad radical entre el saber antropológico y el saber indígena. Sin embargo, debemos tomar consciencia de que por mucho que el/la antropólogo/a intente separarlos, ambos conocimientos deben complementarse. Es decir, los conocimientos también deben servir a los intereses sociales, económicos o políticos de los sujetos de estudio, informantes clave, etc. Si el/la antropólogo/a aprende la lengua o la manera de expresarse de una sociedad para extraer información, también debería servirse de estos códigos para compartir su visión y conocimientos al concluir el trabajo.

Los acuerdos, autorizaciones o permisos con las autoridades indígenas, además de reglamentar el comportamiento del/a investigador/a en las comunidades, deben servir para que el o la investigador/a les entregue su trabajo. Una tesis doctoral no sólo debe pertenece a la academia o servir para obtener un título. Si sabemos hablar con nuestros "sujetos de estudio" para obtener datos, también deberíamos saber hacerlo para compartir nuestros pensamientos con ellos. Mi experiencia confirma que si durante la investigación se entregan datos preliminares ${ }^{7}$ al pueblo, a las autoridades o a los intelectuales locales, la calidad del trabajo mejora considerablemente. Pues nuestras ideas son sometidas a la atenta, experta y crítica mirada de quienes supervisan día a día nuestro trabajo en el campo.

\footnotetext{
${ }^{7}$ Estos resultados, concretamente se trata de un inventario sobre los recursos marinos del sector de Gardi, un censo de ONG kunas y varios artículos académicos, ya han sido utilizados por las autoridades tradicionales para impulsar proyectos de desarrollo sostenible y turismo en la comarca, así como para promover una mayor coordinación de los agentes de desarrollo.
} 


\section{perifèria}

Número 6, julio 2007

www.periferia. name

Después de casi tres años sin pisar la realidad que ha marcado el inicio de este largo camino, pronto volveré a Kuna Yala para entregar la tesis de doctorado e iniciar una nueva etapa de investigación. Una etapa en la que podré valorar el impacto de una investigación realizada desde el respeto y la implicación con un pueblo exigente y autónomo. Una etapa que me permitirá tomar un igar que todavía no sé muy bien donde me llevará. Continuará.

\section{Bibliografía}

Archibold, Guillermo (1993). “PEMASKY en Kuna Yala: Protegiendo a la madre Tierra... y a sus hijos". Archibold, G., Heckadon, S. et al. Hacia una Centroamérica Verde. Seis casos de conservación integrada. DEI, San J osé de Costa Rica.

Congreso General Kuna (2001). Anmar igar. Normas kunas. Panamá.

Fassin, Eric (2007) "L'engagement de l'anthropologue - usages de l'anthropologie". Assises de l'ethnologie et l'anthropologie en France, http://assisesethno.org/spip. php?article49 (última consulta: 14-5-2007).

Tice, Karen E. (1995). Kuna Crafts, Gender, and the Global Economy. University of Texas Press, Austin. 